\title{
Protein extraction protocol from Musa sp. shoots and roots tissue for non-reducing one dimensional SDS-PAGE analysis
}

\begin{abstract}
Background: Protein extraction from plant tissues is a great challenge since they contain low amount of proteins and rich in proteases, secondary metabolites and oxidative enzymes. Besides, dealing with limited amount of starting material would pose another challenge in getting high protein yield. The aim of this study is to determine the best protein extraction protocol for recalcitrant tissues of banana (Musa sp.) specific for non-reducing one dimensional SDS-PAGE analysis. Methods: Three protein extraction protocols were compared which include TCA-acetone, phenol and phosphate buffer saline (PBS) using small amount of starting material. Modifications were done to each protocol to suit non-reducing SDS-PAGE analysis and subsequent downstream processes. Results: Of the three protocols compared, TCA-acetone protocol gave the highest yield and quality of protein extract from only a small amount of starting material based on protein quantification and non-reducing one dimensional SDS-PAGE analysis compared to phenol and phosphate buffer saline (PBS) protocol. Conclusion: Even though various protein extraction protocols have been developed to date, there is no one-size-fits-all when it comes to different types of plant tissues from varieties of plant species. TCA-acetone protocol was found to be most effective in extracting protein from banana shoot and root tissues even for small amount of starting material in absence of reducing agent.
\end{abstract}

Keyword: Protein extraction; Recalcitrant tissue; Musa sp.; Non-reducing SDS-PAGE; TCA-acetone protocol; Reducing agents 